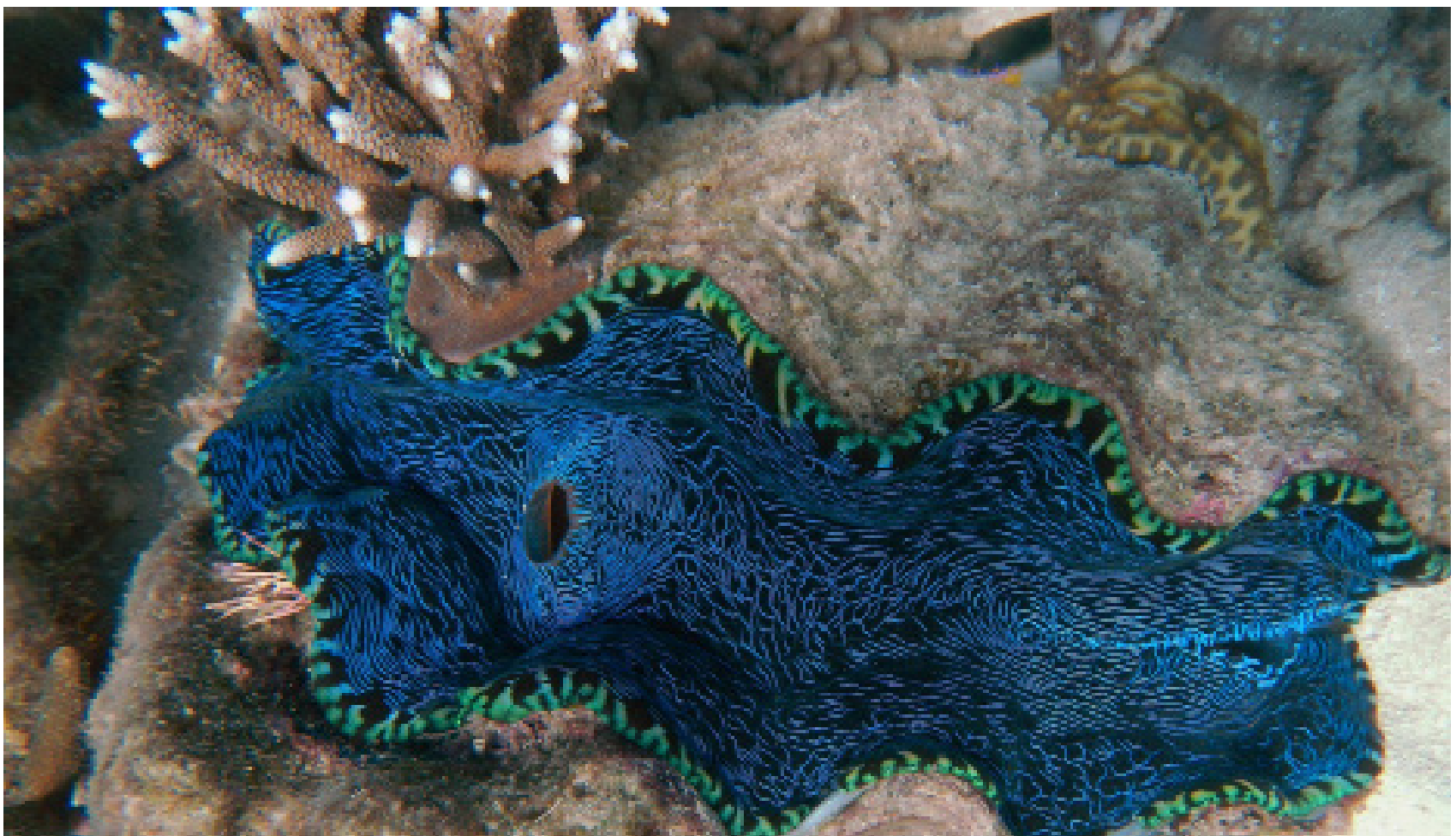

\title{
Pesca sostenible: contribución del capital social en la reconversión laboral para la conservación de la almeja polymesoda sólida
}

\section{Sustainable fishing: contribution of social capital in the reconversion of labour for the conservation of the solid polymesoda clam}

David Eduardo Borge-Donado

Magíster en Desarrollo social, Economista, dborgedonado@ gmail.com, https://orcid.org//0000-0002-6952-3315, Director Situr Atlántico, Barranquilla, Colombia

\section{Ernesto José Cortez-Soto}

Magister en Desarrollo Social, Ernesto.cortez@uniminuto.edu.co, https://orcid.org/0000-0003-2329-0912, Corporación Universitaria Minuto de Dios, Santa Marta, Colombia
Katine Buelvas-Sanjuan

Magíster en Desarrollo Social, katybuelvas@hotmail.com, https://orcid.org/0000-0002-4283-2476, Universidad del Norte Barranquilla, Colombia

David Díaz-Florian

Magíster en Desarrollo Social, ddiaz@uninorte.edu.co, https://orcid.org/0000-0001-9843-7056, Universidad del Norte Barranquilla, Colombia 
Resumen

El propósito de esta investigación fue analizar la pesca o captura de la almeja Polymesoda sólida, de las ciénagas del Vía Parque Isla Salamanca, zona declarada Área Protegida (AP) en la cual se prohíbe la extracción de la almeja. Esta especie está en peligro de extinción por la captura excesiva de aprovechadores que no son personas descendientes de comunidades locales o nativas sino invasores que, pese a la declaración del lugar como Área Protegida que prohíbe la extracción de la almeja, continúan realizando esta actividad ilegal que ya cumple casi 30 años. Los resultados del juego de cooperación, censo y la aplicación de una encuesta semiestructurada en el marco de un taller, confirman en cierto grado lo que se pudo observar en varios días de observación participante de la actividad extractiva: que en el grupo se ha construido un cierto grado de cooperación sin la confianza suficiente como la que se requiere para garantizar el éxito del proyecto alternativo de ecoturismo que les ofrece la autoridad del parque a cambio de suspender la actividad actual. En consecuencia, el problema objeto de la investigación, se concentra en el análisis descriptivo y cualitativo de la información recolectada sobre los dos tipos de relaciones, - las de la economía informal de la almeja, y las de capital social que se articulan con la primera.

Palabras clave: Pesca, Ecoturismo, Capital social, Conservación.

\section{Abstract}

This investigation applies to the case of the fishing or capture of the solid Polymesoda clam, from the swamps of the Vía Parque Isla Salamanca, an area declared a Protected Area (PA) in which the extraction of the clam is prohibited. This species is in danger of extinction due to the excessive capture of landowners who are not descendants of local or native communities but invaders who, despite the declaration of the place as a Protected Area that prohibits the extraction of the clam, continue carrying out this illegal activity that $\mathrm{He}$ is almost 30 years old. The results of the cooperative game, census and the application of a semi-structured survey in the framework of a workshop, confirm to some degree what could be observed in several days of participant observation of the extractive activity: that in the group a Some degree of cooperation without the sufficient confidence that is required to guarantee the success of the alternative ecotourism project offered by the park authority in exchange for suspending the current activity. Consequently, the problem that is the object of the research focuses on the descriptive and qualitative analysis of the information collected on the two types of relationships, - those of the informal economy of the clam, and those of social capital that are articulated with the first.

Keywords: Fishing, Ecotourism, Social Capital, Conservation. 
Introducción

La biodiversidad de Colombia como país, todavía está lejos de aprovecharse económica y socialmente. La biodiversidad es un capital natural cuya conservación exige un esfuerzo por mantener o conservar la vida silvestre aislada de los embates territoriales de la agricultura mecanizada y de la expansión no planificada de la vida urbana. La anterior caracterización aplica al caso de la pesca o captura de la almeja Polymesoda sólida, de las ciénagas del Vía Parque Isla Salamanca, zona declarada Área Protegida (AP) en la cual se prohíbe la extracción de la almeja.

Esta especie está en peligro de extinción por la captura excesiva de aprovechadores que no son personas descendientes de comunidades locales o nativas sino invasores que, pese a la declaración del lugar como Área Protegida que prohíbe la extracción de la almeja, continúan realizando esta actividad ilegal que ya cumple casi 30 años. La gravedad del problema de comunes que se ha configurado con la excesiva pesca de almeja se manifiesta en el hecho de que, en el inicio, la almeja se extraía de tres ciénagas y hoy sólo se extrae de una, y esto se debe al agotamiento de la especie en las otras dos. Los "pescadores" oriundos de distintos municipios de la región Caribe y de la ciudad de Barranquilla en su mayoría, han podido incrementar sus niveles de extracción sin otro impedimento que su propio esfuerzo pesquero, como si la institución de Área Protegida no existiera en esos lugares.

Los resultados del juego de cooperación, censo y la aplicación de una encuesta semiestructurada en el marco de un taller, confirman en cierto grado lo que se pudo observar en varios días de observación participante de la actividad extractiva: que en el grupo se ha construido un cierto grado de cooperación sin la confianza suficiente como la que se requiere para garantizar el éxito del estudio alternativo de ecoturismo que les ofrece la autoridad del parque a cambio de suspender la actividad actual. En consecuencia, el problema objeto de la investigación, se concentra en el análisis descriptivo y cualitativo de la información recolectada sobre los dos tipos de relaciones, - las de la economía informal de la almeja, y las de capital social que se articulan con la primera.

La anterior caracterización aplica al caso de la pesca o captura de la almeja Polymesoda sólida, de las ciénagas del Vía Parque Isla Salamanca, zona declaradaÁreaProtegida(AP) en la cual se prohíbe la extracción de la almeja. Esta especie está en peligro de extinción por la captura excesiva de aprovechadores que no son personas descendientes de comunidades locales o nativas sino invasores que, pese a la declaración del lugar como Área Protegida que prohíbe la extracción de la almeja, continúan realizando esta actividad ilegal que ya cumple casi 30 años. Los fundamentos de ley que determinan la ilegalidad de la actividad pesquera por parte de los almejeros, se puede constatar en las observancias del [1]. donde se establece: "que el uso, manejo y destinación de dichas áreas está sujeto de forma estricta a unas finalidades específicas de conservación, perpetuación en estado natural de muestras, y protección de diferentes fenómenos naturales y culturales" [2]. .Así mismo se pregona en el mencionado Decreto ley, que "las actividades permitidas en el área de parques naturales son, exclusivamente, conservación, investigación, educación, recreación, cultura, recuperación y control.

La gravedad del problema de comunes que se ha configurado con la excesiva pesca de almeja se manifiesta en el hecho de que, en el inicio, la almeja se extraía de tres ciénagas y hoy sólo se extrae de una, y esto se debe al agotamiento de la especie en las otras dos. Los "pescadores" oriundos de distintos municipios de la región 
Pesca sostenible: contribución del capital social en la reconversión laboral para la conservación de la almeja polymesoda sólida

\section{0}

Caribe y de la ciudad de Barranquilla en su mayoría, han podido incrementar sus niveles de extracción sin otro impedimento que su propio esfuerzo pesquero, como si la institución de Área Protegida no existiera en esos lugares. La actual administración del Parque les ha propuesto una solución negociada que consiste en abandonar la extracción de almeja a cambio de dedicarse a la actividad del ecoturismo para lo cual se les apoyará con la capacitación y entrenamientos necesarios, y la dotación de lanchas y motores fuera de borda para la prestación del servicio. A la fecha, la administración del Parque no ha fijado fecha o plazo perentorio para el recambio de actividad y por ello, se percibe entre los almejeros un comportamiento tranquilo, de normalidad, en la faena diaria de extracción de la almeja.

La falta de presión policiva en las últimas dos décadas, y la convicción que muestran los invasores de que la administración no recurrirá al control policial, les ha permitido en medio de un clima de estabilidad, construir algunas relaciones de cooperación, aunque de mínima confianza, que han incidido en la mejora de la calidad del producto y del ingreso de todos. El objetivo de la presente investigación utiliza los resultados de un ejercicio de Juego de Cooperación para examinar qué tanta cooperación y confianza están dispuestos a aportar para el éxito de la transición desde pescadores ilegales privados o independientes, a operadores ecoturísticos legales funcionando como un colectivo emprendedor en el mismo espacio del AP.

En este trabajo, se trata de establecer si la cooperación incipiente que observa en la realidad de la captura de almejas y en el juego de cooperación llevado a cabo con 19 de los 49 almejeros, según censo de 2016, es suficiente y promisoria como para garantizar que todos cooperarán y fortalecerán lazos de confianza cuando les toque cambiar de actividad, que a diferencia de la actual, es legal y formal, y requiere de alta responsabilidad para su manejo administrativo trasparente y que represente ingresos remunerativos para todos.

Preguntas orientadoras

¿Podrá ser el Capital Social Adquirido una alternativa de transformación hacia el ecoturismo para un grupo de almejeros del área protegida Parque Natural Nacional Vía Isla Parque de Salamanca en el Departamento del Magdalena?

- ¿Cómo determinar los componentes del capital social adquirido durante la actividad de captura de almejas, que posibiliten el diseño de una propuesta de alternativa de reconversión laboral hacia el ecoturismo, entre los almejeros ilegales ubicados en el Parque Natural Nacional Isla Salamanca?

- ¿Qué técnica de la investigación orienta la identificación de las características sociales, económicas y culturales del grupo de almejeros ubicados en el Parque Natural Nacional Isla Salamanca?

- ¿Cómo se materializa o evidencia la problemática medio ambiental depredadora que viene sucediendo como consecuencia de la actividad de los almejeros en la Isla Salamanca, Área Protegida donde se prohíbe la extracción de la almeja?

- ¿Qué oportunidades se pueden aprovechar de la descripción y caracterización enmarcadas en las relaciones de cooperación y confianza que sean percibidas como capital social de los almejeros, para facilitar su transición hacia el manejo colectivo o cooperativo de un emprendimiento en ecoturismo?

Materiales y métodos

La investigación se realizó bajo el enfoque. Abordando las experiencias, costumbres, 
formas de vida, carencias y/o necesidades de la población objeto de estudio.

El tipo de investigación fue descriptivaexplicativa, el que cual permitió describir y analizar las características de la población de almejeros que se dedican a la extracción en VIPIS para analizar ciertos comportamientos y concluir si el capital social adquirido en su diario vivir es suficiente para poder llevar a cabo una transformación laboral.

Dentro de los 48 almejeros identificados y caracterizados por INVEMAR como la población de almejeros, 19 decidieron acogerse a la propuesta de VIPIS por lo cual se convierten en la muestra de este estudio de investigación; teniendo un tamaño de muestra de 19 personas, junto a ellos se realizó una constante recopilación de información a través de la observación en la cual 3 fases de recopilación de información:

- Censo con los almejeros, buscando caracterizarlos socioeconómicamente

- Entrevista semiestructurada buscando conocer su actividad a fondo y su percepción sobre el ecoturismo

- Juego de cooperación buscando identificar componentes de capital social.

Además de la constante observación en sus actividades diarias, buscando encontrar indicios de cooperación y confianza.

Resultados

Características socioeconómicas. El perfil socioeconómico de los almejeros que pescan en el sector occidental del Parque y que accedieron al trabajo de observación, taller y encuesta para esta investigación censados para la Administración del Parque, se presenta a continuación:
Procedencia, lugar de nacimiento y residencia. Con respecto al lugar de procedencia, el $58 \%$ son provenientes del Atlántico (11), Del Dpto. de Magdalena procede el 32\% (6), Bolívar $5 \%$ (1) y Córdoba 5\% (1). La procedencia del Departamento de Atlántico se distribuye así: Barranquilla con el $60 \%$ (6) de la procedencia, seguido de Malambo con $27 \%$ (3) y Soledad con 18\% (2). De los de Magdalena Pueblo Viejo registra $50 \%$ (3) y Palermo 33\% (2).

En cuanto al lugar de residencia, 6 viven en el municipio de Soledad, 5 en Palermo, 4 en Malambo, 3 en Barranquilla y 1 en Sitionuevo.

Características Etáreas. Del total, 8 pescadores tienen entre 31 y 40 años (42\%) cinco de ellos (21\%) tienen entre 20 y 30 años y los 6 restantes tienen edades que oscilan entre 41 y 50 años. Esto permite inferir sobre su alto potencial laboral y sobre el largo tiempo que continuarían depredando los bancos de almeja si el problema no se resuelve.

Edad de inicio del aprovechamiento de la almeja en la VIPIS. EI 78,9\% de los pescadores encuestados tenía entre 11 y 20 años de edad cuando iniciaron la actividad; $15.7 \%$ tenía entre 21 a 30 años; $5,2 \%$ restante tenía entre 8 y 10 años.

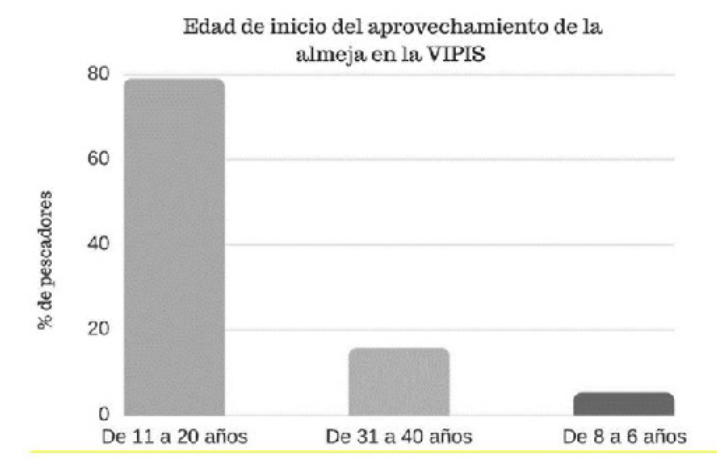

Gráfico 1. Edad de inicio Del aprovechamiento de la almeja en la VIPIS. 
Pesca sostenible: contribución del capital social en la reconversión laboral para la conservación de la almeja polymesoda sólida

\section{2}

Nivel Educativo. El $36 \%$ de los pescadores manifestaron haber alcanzado algún grado de educación básica primaria, el $31 \%$ señaló haber alcanzado algún grado de básica secundaria; mientras que el $26 \%$ restante expresó no haber cursado grado escolar alguno. Uno de ellos con secundaria completa, realizó un curso de entrenamiento (nivel SENA) en mantenimiento de computadores. Este pescador se destaca por exhibir un cierto nivel importante de liderazgo.

Estado Civil. El $63 \%$ de los pescadores vive en unión libre, mientras que el $21 \%$ y el $10 \%$ indicaron que están casados y divorciados respectivamente.

Personas a cargo. En total 98 personas (familiares) dependen directamente de los 19 pescadores. El $57.8 \%$ de los pescadores pertenecen a un núcleo familiar entre 4 a 6 personas. El $21 \%$ afirmó tener de 1 a 3 personas a su cargo, mientras que otro $21 \%$ tiene a su cargo más de siete (7) personas. Del total de personas a cargo, $98(40,8 \%)$ corresponde a menores de 18 años, el 14,28\% corresponde a personas entre 19 a 39 años, el $30,63 \%$ a personas entre los 31 y 40 años, el $3.5 \%$ a personas entre los 41 a 50 años y el $14,28 \%$ corresponde a personas mayores de 51 años.

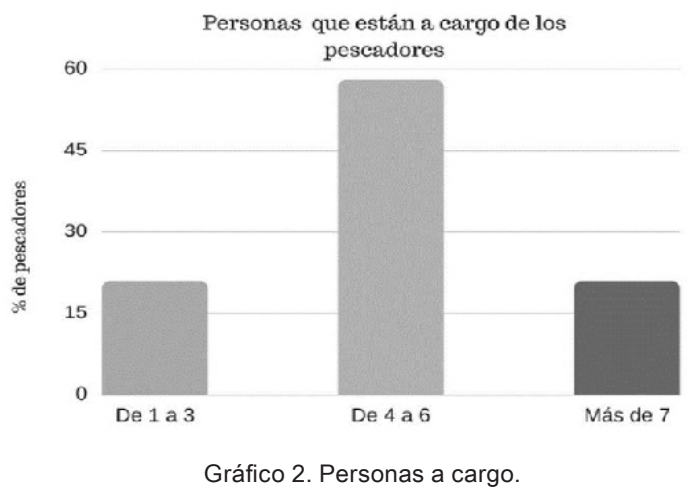

Ingresos y gastos mensuales. El $100 \%$ de los pescadores encuestados manifestó que su gasto promedio mensual en la extracción de la almeja es menos de un salario mínimo mensual legal vigente, que oscila entre $\$ 150.000$ y $\$ 450.000$, un rango que refleja una alta variabilidad que se debe a que los implementos de pesca, aunque rudimentarios - artesanales varían en su grado de manufactura o elaboración. En cuanto a las ganancias o ingresos netos luego de la venta, $57,8 \%$ afirmó obtener más de dos salarios mínimos mensuales, mientras que $36.8 \%$ aseguró percibir entre uno y dos salarios mínimos legales vigentes; $5,2 \%$ afirmó percibir menos de un salario mínimo legal vigente.

Confianza. Para la confianza y cooperación en el caso del grupo de almejeros, las declaraciones durante el taller de juegos económicos muestran que se trata de un grupo con integrantes que proceden de Barranquilla y de otras cabeceras municipales de vida rural. A diferencia de las comunidades de pescadores de la Ciénaga Grande, los almejeros no conforman una comunidad tradicional, llegan por la mañana y se van por la tarde, de regreso a sus respectivas comunidades de los lugares donde residen. No se detectaron relaciones sólidas de confianza ni de cooperación, pero sí unas relaciones incipientes que son válidas como prueba del comienzo de la construcción de confianza. A continuación, una lista de evidencias de cooperación:

- Los participantes manifestaron que colaboran económicamente entre sí para financiar el transporte diario; manifestaron que de manera espontánea comparten una rutina colectiva de tomar el transporte fluvial de ida y regreso todos los días de faena.

- Las labores de desconche de la almeja se hacían individualmente y de forma manual muy rudimentaria. Desde hace varios años, supieron que, al pasar la almeja por agua caliente, las conchas se abren fácilmente lo cual facilita la separación y posterior empaque del producto. Uno de los pescadores proveyó 
la marmita y desde entonces, los almejeros se rotan en grupos de dos para la preparación diaria del fuego, el control del tiempo de precocción de la almeja, y su recuperación y empaque.

- Otro caso de una incipiente cooperación espontánea es el uso colectivo del bote de propiedad de uno de ellos, que lo utilizan para almacenar lo que cada uno extrae durante la faena del día. Luego, el bote transporta la almeja al lugar de tierra firme donde se instala el fuego y la marmita para desconchar por cocción las cantidades extraídas de cada uno. Desde que existe esta cooperación, no se reportan casos de conflicto interpersonal durante el proceso. Cada uno cuida lo suyo al momento de empacar lo producido para su venta al primer intermediario. Todos reconocen que la cooperación en el proceso de desconche, mejoró la calidad de la almeja en cuanto a su presentación y sanidad, y que eso ha beneficiado a todos por igual.

El problema de la confianza ha dado lugar a una amplia e importante literatura ubicada en diversos contextos teóricos y disciplinarios. Considerada como ingrediente principal del capital social, la confianza se ha convertido en una variable indispensable para explicar el funcionamiento de la democracia o el desempeño económico.

Aun así, cabe resaltar que después de haberse realizado el juego de cooperación, los almejeros entre si manifestaron no confiar del todo en los demás. Las expectativas que mostraron sobre las acciones de los demás fueron con patrones de desconfianza, al mostrar que no fueron capaces de cooperar del todo debido a que no estaban seguros de que los otros lo harían. La importancia de estas expectativas se mostró en el curso de dicho juego de cooperación ya que tenían que hacer esa elección antes de que pueda evaluar las acciones de los otros del [3].
Si bien hubo patrones de desconfianza, los almejeros mostraron que si hubieran conocido su grupo y además si este grupo hubiera estado conformado por los almejeros de su red social ya sea sanguínea o vecinos de casa, la relación de confianza hubiera sido mucho mayor y estuvieran más tranquilos que la contraparte no abusará de la persona que desea cooperar del [4]..Dentro de estos vínculos de confianza por amistad, aparte de la consanguinidad y vecindario notamos patrones comunes como la búsqueda de un líder, o fortalezas afines.

En la comunidad de almejeros especialmente los más estudiados y quienes han realizado otras actividades distintas a la almejería, lideran grupos —o "cuasi grupos" [5]. menos definidos- dentro del grupo total de almejeros, que les sirven para acumular tanto bienestar material como prestigio. De esta manera, por un lado, la reciprocidad vertical y el faccionalismo son intrínsecos a las redes interpersonales de la comunidad de almejeros; pero por otro, el liderazgo basado en esta reciprocidad vertical puede ser la base de la cooperación y la ampliación de la organización más allá de su organización. Estos líderes de los cuasi grupos son los empresarios del capital social, y gestionan los recursos humanos de sus parientes y vecinos, ante los cuales tienen obligaciones y deben rendir cuentas; muchas de las actividades grupales externas son motivadas por ellos, así como muchos de los cambios para tecnificar la actividad del almejero- la inclusión de elementos y técnicas para facilitar la extracción, así como la simplificación del transporte desde sus casas hasta el sitio de extracción-.

Entre las frases que muestran una sensación de desconfianza hacia el proceso se encuentran "si las cosas no salen porque hasta ahora hemos visto muchas promesas, pues uno volverá a la ciénaga porque no sabe hacer otra cosa" y "pero yo hasta ahora no veo nada, no 
Pesca sostenible: contribución del capital social en la reconversión laboral para la conservación de la almeja polymesoda sólida

\section{4}

he visto que Parques nos de nada, prometen y prometen nos hacen venir y ya". Dentro de la desconfianza que muestran mencionaron frases como "Ninguno de nosotros puede ser administrador, necesitamos contratar alguien de afuera", "Había que aportar, pero antes de aportar yo tenía que asegurar algo, si hubiera sabido quienes eran mis compañeros hay quizá hubiera pensado bien que hacer, pero sin saber difícil, hay unos que son vivos", "Había que aportar, pero antes de aportar yo tenía que asegurar algo, si hubiera sabido quienes eran mis compañeros hay quizá hubiera pensado bien que hacer, pero sin saber difícil, hay unos que son vivos", "Si el juego hubiera sido con 1.000 .000 prefiero asegurarme, porque es más dinero, así que me hubiera aguantado mucho más, porque con más dinero podría asegurar mis necesidades básicas. Aunque evidentemente el vivo va a jugar pensando, si no juego nada y los otros juegan todo, la cantidad que voy a ganar es considerable".

La solidaridad para los almejeros tiene un valor importante. Todavía existen formas de cooperar con los demás. La ayuda se constituye en una característica esencial y la diferencia de otros grupos de pescadores.

En este orden de ideas, Cohen y Prusak [6]. definen el capital social como la "totalidad de las relaciones activas entre las personas: la confianza, la comprensión mutua, los comportamientos y valores compartidos que unen a los miembros de una comunidad y las redes humanas que hacen posible la acción cooperativa", la cual coincide con lo observado en la comunidad de almejeros.

Los almejeros depositan su mayor confianza, en primer lugar en los líderes, quienes son designados por consenso y tienen cierto grado de escolaridad y perfil de liderazgo, para gestionar decisiones que contribuyan a responder a las necesidades del grupo de almejeros, en ellos además de la confianza, se sienten altamente representados, en segundo lugar en las personas mayores de edad, quienes gozan del respeto de los miembros de la comunidad y en tercer lugar en los compañeros que viven en su misma zona municipio, corregimiento o barrio- puesto que con estos tienden a compartir actividades conjuntas extras tan simples como la toma del transporte público que los lleva al sitio de salida a pescar.

Existe reciprocidad como un comportamiento que hace parte del diario vivir en un conjunto de almejeros que llevan años compartiendo la actividad y que va más allá de la expectativa de ser correspondido hasta llegar a comportamientos como la solidaridad y el compromiso de ayudar a quienes comparten las mismas actividades.

Retomando la afirmación de [7] la reciprocidad es una regla fundamental de la vida, y teniendo en cuenta los resultados de esta investigación, la afirmación gana mayor veracidad en las condiciones de comunidad, ya que para ellos el intercambio de favores con sus vecinos y la ayuda mutua es lo que les ha permitido sobreponerse a los problemas. Es decir, la reciprocidad es básica para el equilibrio y sostenimiento de un grupo o comunidad en situaciones críticas.

En primer lugar, se encontró que la ayuda que brinda cada almejero hacia otro está dirigida principalmente a solucionar los problemas y necesidades básicas relacionadas con enfermedad, transporte y alimentación. EI intercambio de favores está relacionado con los conocimientos y habilidades que posee cada uno y la posibilidad de tener algo que el otro no tiene y necesita en el momento preciso; por ejemplo, es muy común el intercambio de herramientas -rastrillo y neveras de icoporasí como suplir en algún momento preciso de la pesca - ayudar a hervir la almeja, o a armar los sacos -. De esta forma, la reciprocidad, 
como afirma [8]. aparece en una situación de carencia para facilitar el uso de algunos recursos que otros no poseen.

En el caso de que algún miembro de la comunidad tenga excepcionalmente un poco más de comida o de dinero, la colaboración que brinda a otro vecino no la da en términos de préstamos sino de regalo, y a pesar de que esperan que el compañero avise sobre la necesidad que tenga, en el momento de contar con más recursos de lo normal lo reparten entre sus compañeros sin que éstos se lo hayan pedido; aspecto que apoya la teoría de [9], quien afirma que la reciprocidad da paso al trato altruista y una conducta prosocial.

Los almejeros no tuvieron incomodidad para hablar sobre la ayuda que solicitan a sus vecinos, encontramos que al pedir o solicitarla lo hacen fundamentalmente por obligaciones de satisfacer necesidades básicas principales de sus hijos. Entre compañeros buscan colaboración en casos de una fuerte necesidad como una enfermedad de un hijo, o cuando no hay comida.

Por otro lado, en ocasiones han recibido la ayuda de compañeros sin haberla solicitado. En esos momentos la ayuda está relacionada con el apoyo y acompañamiento a la persona en un caso de emergencia, con el ofrecimiento de comida o sencillamente la invitación a colaborar en un momento del día al trabajo que estén realizando dentro de la pesca.

Debido al compartir diario en sus actividades, consideran al otro almejero como un familiar cercano y que puede socorrerlos en un caso de emergencia.

Ayudarse entre almejeros es casi que una norma común. El hecho de encontrarse en condiciones parecidas de trabajo informal, hace que la expectativa de ayuda y disposición a colaborar sea mayor. Esto apoya lo expresado por [10], quien afirma que la reciprocidad rige con más fuerza en las interacciones con nuestros iguales.

Los almejeros valoran la interacción y cooperatividad entre ellos, de hecho, se da una cooperación constante, debido a que cada uno solicita y presta ayuda según las posibles necesidades de otros. Así, si uno de ellos dejó tiene un dolor de brazo y no puede batir las almejas mientras la cocinan, se ofrece a armar los sacos de almeja de su compañero - que es una actividad de menos esfuerzo - mientras este bate sus almejas.

Otro ejemplo puede notarse cuando un almejero no tiene completa la plata del bus y se la pide prestado a un compañero que en alguna o varias ocasiones le han solicitado que le preste utensilios de pesca cuando los ha dejado.

De acuerdo con esto y apoyado en la teoría de [11] se puede describir la relación de cooperación entre los almejeros como un intercambio de bienes, valores y servicios que les facilita el sostenimiento diario frente a sus necesidades. Los almejeros manifiestan una cooperación constante, pero podríamos decir limitada, debido a que el mayor número de intercambios se produce en grupos pequeños de acuerdo con la cercanía física.

Aspectos sociales. Teniendo en cuenta que la almeja ha estado sujeta a explotación ilegal y que a principios de los años 70 se distribuía en la Ciénaga Grande de Santa Marta (CGSM) y Complejo Pajarales [12], pero no volvió a ser registrada posteriormente [13], se puede afirmar que el Sector Occidental del Vía Parque Isla de Salamanca constituye relicto de esta especie en la ecorregión CGSM [14]. En este sentido, se plantea la necesidad de hacer un trabajo articulado entre las entidades que tienen competencia en el tema en los departamentos del Atlántico y Magdalena. 
Pesca sostenible: contribución del capital social en la reconversión laboral para la conservación de la almeja polymesoda sólida

\section{6}

Con mayor participación y liderazgo del departamento del Atlántico a través de su Gobernación; esto en atención a qué el $93.75 \%$ de los pescadores que hacen la extracción de almeja en la VIPIS provienen de municipios que hacen parte de este departamento.

Considerando que un pescador artesanal típico del Caribe colombiano será un hombre maduro, de mediana edad (unos 48 años) y con una experiencia de algo más de 27 años [15] es posible inferir que la edad de inicio en la actividad pesquera estaría cercana a los 21 años, aspecto que coincide con los rangos de edades observadas en el $62 \%$ de los pescadores encuestados, quienes se encuentran entre 20 y 40 años de edad.

En las áreas protegidas del Caribe se ha evidenciado que el $50.2 \%$ de los pescadores que hacen uso y aprovechamiento es encuentran en Unión libre [16], lo cual coincide con lo encontrado por la Autoridad Nacional de Acuacultura y Pesca (AUNAP) y la Universidad del Magdalena a través de la implementación del Sistema Estadístico Pesquero de Colombia (SEPEC) quienes reportaron que el $64 \%$ de los pescadores del país se encuentran en estado civil: Unión libre Lo anterior es similar a lo observado en el presente análisis, donde el $63 \%$ vive en unión libre.

De manera general [17] (en evaluación), identificaron que por encima del $70 \%$ de los pescadores que hacen uso y aprovechamiento en las áreas protegidas no saben leer ni escribir. Situación similar a la observada durante la aplicación de la encuesta a pescadores de almeja, donde el $30 \%$ indicó que no saben leer ni escribir y el $36 \%$ manifestó haber alcanzado algún grado de la primaria.

El núcleo familiar de los pescadores en su mayoría (más del 78\%) está compuesto por 4 a 7 personas que están a su cargo. Este aspecto guarda relación con lo reportado por la AUNAP y UNIMAG en el SEPEC.

En total 99 personas dependen económicamente de la extracción de almeja, de los cuales 19 son los pescadores y 80 miembros de sus familias. De éstas últimas personas más del $53 \%$ corresponden a menores de edad. El $30 \%$ de las personas que dependen económicamente de los pescadores no poseen algún grado de escolaridad, lo cual pone de manifiesto la necesidad de que se generen opciones que permitan que estas personas sean atendidas.

Así mismo, es necesario revisar la posibilidad de que se generen oportunidades para que se vinculen a los pescadores y sus familias en proyectos de adjudicación de vivienda. Esto en razón a que el $52 \%$ de los pescadores manifestaron que habitan en vivienda arrendada con mensualidades que oscilan entre \$150.000 - \$200.000, mientras que otro $37 \%$ habitan en viviendas familiares donde si bien no pagan un arriendo directo dan aportes de aproximadamente 50.000 de servicios.

Según lo registrado por la AUNAP y la UNIMAG a través del SPEC el $72 \%$ de los pescadores encuestados durante la caracterización nacional opinaron no tener servicio de salud. Situación que es vidente en los pescadores de almeja, quienes en un $36.5 \%$ indicaron que no cuentan con ninguna vinculación al Régimen Subsidiado por Estado colombiano, ni ningún otro sistema.

El $56.25 \%$ de los pescadores inició la actividad de extracción de almeja entre los 11 y 20 años de edad. Lo cual, contrastado con la edad promedio (40) de los pescadores, permite inferir sobre su potencial productivo. Aspecto que debe ser tenido en cuenta por las entidades encargadas de atender el problema social, para evaluar las posibles opciones de alternativas económicas que puedan formular 
en beneficio de los pescadores, como estrategia para erradicar la presión sobre el recurso almeja en el área protegida.

Por otra parte, a través del presente análisis, se pudo determinar que $81.2 \%$ de los pescadores de almeja realizan su actividad durante los 12 meses del año y de 20 a 26 día por mes. Lo cual, es concluyente sobre el nivel de presión al que se encuentra sometida P. solida. Este aspecto es coherente con lo reportado por [18] quienes reportaron para esa época una tasa de explotación de 0.71 , siendo superior a la óptima de explotación (0.41). Es decir, indicando que el recurso era sobre-explotado. Aspecto que ha sido ratificado por INVEMAR a través de documentos técnicos, donde manifiesta que el recurso se encuentra en riesgo de sobre explotación por reclutamiento y crecimiento y lo más grave riesgo de colapso [19].

- Mientras que la literatura de capital social como elemento clave de la acción colectiva necesaria para frenar un problema de comunes abunda en casos de comunidades que aprovechan el recurso de manera insostenible, en este caso lo novedoso es que no se trata de una comunidad en el sentido tradicional cultural sino de invasores que han aprovechado la debilidad de las autoridades para hacer cumplir las normas que rigen un Área Protegida. Este grupo, que no comunidad, ha aprovechado la debilidad o ausencia de control para extraer una especie endémica, lo cual configura una falla de gobierno.

- A lo largo de más de 30 años este grupo mantiene la desconfianza propia de actores ilegales, pero han demostrado que cooperan para procesar el producto diario y mejorar los estándares de calidad e higiene antes de llevarlos al mercado. Esto hace eco de artículos que muestran que, en la acción colectiva, la confianza no es sine qua non de la cooperación.
- El juego de cooperación confirma la situación de cooperación sin confianza que se evidenció en la actividad de observación participante realizada antes del juego. Es posible que las muestras de cooperación estén determinadas por su uniforme comportamiento antisocial colectivo como invasores de la ciénaga y practicantes de una actividad ilegal. Nada permite predecir que este comportamiento quizás defensivo se reproduzca en la actividad formal y administrativamente más exigente como lo es el emprendimiento ecoturístico.

\section{Referencias}

[1] B. Aylward, K. Allen, J. Echeverría y J. Tosi, "Sustainable ecotourism in Costa Rica: the Monteverde Cloud Forest Preserve", Biodiversity \& Conservation, vol. 5, pp. 315343, 1996

[2] J. Belsky, "Misrepresenting communities: the politics of community-based rural ecotourism", Rural Sociological Society, 1999

[3] F. Block, "Karl Polanyi and the writing of The Great Transformation", Theory and Society, vol. 32, pp 275-306, 2003

[4] P. Bourdieu, Raisons pratiques, Sur la theorie de laction, Editions du Seuil, Paris, 2001

[5] K. Brandon et al. People and parks: linking protected area management with local communities. World Bank. Washington D.C., Estados Unidos, 1992

[6] K. Brandon, Moving beyond Integrated Conservation and Development Projects (ICDPs) to achieve biodiversity conservation. CABI, 2001.

[7] J. Cárdenas, Dilemas de lo colectivo. Instituciones, pobreza y cooperación en el manejo local de los recursos de uso común. 
Pesca sostenible: contribución del capital social en la reconversión laboral para la conservación de la almeja polymesoda sólida

28

CEDE, Universidad de los Andes, Bogotá, Colombia, 2009.

[8] H. Ceballos-Lascuráin,Tourism, ecotourism and protected areas: The state of nature-based tourism around the world and guideline for its development, IUCN, Cambridge, Reino Unido. 1996

[9] J. Coleman, Social capital in the creation of human Capital" in, Partha Dasgupta and ismaail Serageldin (Eds), Social capital, a multifaceteds prospectiva, World Bank, Washington, 2000

[10] R. Cornes et al., "On commons and tragedies", American Economic Review, vol. 73, no. 4, 1983

[11] R. Cornes et al., "The commons and the optimal number of firms", The Quaterly Journal of Economics, vol. 101, no. 3, 1986

[12] R. Cornes et al., The theory of externalities, public goods and club goods, Cambridge University Press, 1996

[13] R.V Cosel, "Moluscos de la región de la Ciénaga Grande de Santa Marta (costa del Caribe de Colombia)", An. Inst. Inv. Mar. Punta Betín, no. $15-16$, pp. 79-370, 1986

[14] M.V De la Hoz, "Distribución, abundancia y aspectos biológicos de la almeja Polymesoda solida Phillippi, 1846 (Bivalvia: Corbiculidae) en la Isla de Salamanca, Caribe colombiano", Tesis MSc, Universidad Nacional de Colombia, Santa Marta, Magdalena, Colombia, 2005

[15] J.M. Díaz y M. Puyana, "Moluscos del Caribe colombiano. Un catálogo ilustrado", Colciencias, Fundación Natura, INVEMAR, Bogotá, 1994

[16] J. Durston, Evaluando Capital Social en Comunidades Campesinas en Chile, ponencia preparada para el XXIII Congreso de Lasa, Washington, 6-8 septiembre Departamento Nacional de Planeación, Política de Bosques. Documento CONPES No. 2834, Departamento Nacional de Planeación: Bogotá, Colombia, 2001

[17] A. Drumm and A. Moore, Ecotourism Development - A Manual for Conservation Planners and Managers. Volume I - An Introduction to Ecotourism Planning, USA: The Nature Conservancy, 2002.

[18] Fundación Marviva, Diagnóstico regional de condiciones y evaluación de las necesidades de capacidades institucionales para la aplicación del Acuerdo de FAO sobre Medidas de Estado Rector de Puerto, en Costa Rica, Panamá y Colombia. Fundación MarViva, 2016

[19] E. González, Ecotourism as a mean for community-based sustainable development: La Congreja National Park case study. Wageningen, Países Bajos, Wageningen University \& Research Center, 2004 